Ann. Biol. anim. Bioch. Biophys., r973, 13 (4), 643-657.

\title{
CONDITIONS DE CLONAGE ET DE CULTURE EN SUSPENSION DE LA SOUCHE BHK 21/13 DE HAMSTER SYRIEN SENSIBILITÉ DES CELLULES AUX INHIBITEURS DU MÉTABOLISME DES ACIDES NUCLÉIQUES
}

\author{
M. CABOCHE \\ avec la collaboration technique de Ginette GiFFARD \\ Laboratoire de Génétique cellulaire. \\ Centre de Recherches de Toulouse, I. N.R.A., \\ B. P. 12, 31320 Castanet Tolosan
}

\begin{abstract}
RÉSUMÉ
Les caractéristiques de la souche $\mathrm{BHK} 2 \mathrm{I} / \mathrm{1} 3$ permettent de l'utiliser facilement comme outil génétique.

Les conditions de culture et de clonage de la souche sont analysées.

En milieu MEM contenant 8 p. Ioo de sérum fœetal bovin dialysé l'efficacité d'étalement est médiocre (I p. IOo). En présence de sérine et d'asparagine une croissance clonale correcte est obtenue (25 p. roo d'efficacité d'étalement). La souche BHK 2I/3I S r 3 adaptée à la culture en suspension prolifère en présence de ces métabolites à des concentrations cellulaires très basses (Io ${ }^{4}$ cellules/ml) avec un temps de doublement de I4 heures. La composition du milieu évolue au cours de la croissance, des quantités importantes d'alanine, de glycine et de proline sont rejetées dans le milieu à faible densité de population. Les cultures confluentes épuisent le milieu en amides. Les conditions optimales de clonage correspondent à un $\mathrm{pH}$ de 7,2 et une température de $37^{\circ} \mathrm{C}$.

Le seuil de résistance de la souche à diverses drogues affectant le métabolisme des acides nucléiques est déterminé (bromodéoxyuridine, azaguanine, aminoptérine).

Le caryotype de la souche $(\bar{n}=43)$ observé après hydrolyse trypsique ménagée est comparé au caryotype de hamster syrien $(\bar{n}=44)$. Toutes les paires de chromosomes sont distinguées par cette méthode.
\end{abstract}

\section{INTRODUCTION}

La mise au point de technique de culture in vitro puis des conditions de clonage de certaines souches de cellules somatiques de mammifères (Puck et al., 1956) permettent d'étudier des mécanismes héréditaires jusque-là inaccessibles par 1'étude 
directe de ces animaux. Par exemple, les mutations affectant le métabolisme intermédiaire, le plus souvent létales ou cachées chez l'animal, peuvent être étudiées in vitro.

Des lignées résistantes à la 8-azaguanine et à la 5-bromodéoxyuridine et affectées dans le métabolisme des bases puriques ou pyrimidiques ont été isolées à partir de la souche BHK 2I/I3 (Mac Pherson et Stocker, I962). Cette souche est donc un outil génétique intéressant. Cependant pour être utilisable en génétique, une lignée cellulaire doit posséder un certain nombre de propriétés :

- Elle doit être facilement clonable afin d'obtenir des lignées provenant d'une cellule isolée.

- Elle doit posséder une hérédité stable, évoluant peu au cours des divisions successives.

- Il est souhaitable enfin que ses conditions de culture rendent son emploi aisé (prolifération rapide dans un milieu de composition simple). La souche BHK $2 \mathrm{I} / \mathrm{I} 3$, isolée à partir du rein de hamster syrien, possède des caractéristiques correspondant assez bien aux deux derniers points de cette description :

Elle se cultive à la fois en tapis et en suspension. Son caryotype est stable et proche de celui de l'animal. Enfin son temps de doublement très court (I2 heures) rend son emploi très aisé en génétique, réduisant les temps de clonage de façon appréciable.

Cependant un problème subsiste au niveau des conditions de clonage. Les cellules sont usuellement cultivées en milieu de base Eagle modifié et complété selon Mac Pherson et STocker (I962). Dans ce milieu l'efficacité d'étalement de la souche ne dépasse pas I p. roo. En outre l'emploi de tryptose et de sérum non dialysé comme complément au milieu synthétique rend difficile l'étude de l'action de certaines drogues sur le métabolisme. Ces drogues entrent en compétition avec des quantités parfois importantes de métabolites dont elles sont les analogues ou les inhibiteurs de synthèse. Dans ces conditions leur effet nocif peut être complètement masqué.

La mise au point de conditions de culture permettant un clonage facile de la souche a été effectuée et ceci dans un milieu dont tous les constituants de bas poids moléculaires sont connus. Ce milieu permet de cultiver les cellules soit en tapis soit en suspension à partir d'une faible densité de population initiale.

A l'aide de ce milieu de culture, le rôle de certains acides aminés dans la croissance à faible densité cellulaire a été mis en évidence. Le setuil de résistance à diverses drogues a été étudié.

\section{MATÉRIEL ET MÉTHODES}

\section{I. - Souches cellulaires}

La souche BHK 2I/13 (Stoker et Mac Pherson, 1964) provient de l'ATCC $\left(\mathrm{CCL}_{10}\right)$. La souche BHK $2 \mathrm{I} / \mathrm{I} 3$ adaptée à la culture en suspension à forte concentration cellulaire (CAPSTICK tt al., I962) et appelée $\mathrm{S}_{3} 3$ provient du Laboratoire de Virologie de Grignon (1).

La présence de PPLO dans les cultures n'a pu être décelée par la méthode de Todaro et al. (197 I). Les souches sont conservées congelées à $-70^{\circ} \mathrm{C}$ dans le MEM contenant en outre $15 \mathrm{p}$. roo de glycérol et $20 \mathrm{p}$. roo de sérum foetal bovin.

(1) Adresse : Laboratoire de Virologie et d'Immunologie, 78 Thiverval-Grignon. 


\section{2. - Produits chimiques}

Les acides aminés, les sucres, le pyruvate de sodium et le Tris employés dans cette étude sont des produits Merck proanalysis. L'hypoxanthine, la thymidine et la 5-bromodéoxyuridine sont des produits Calbiochem A grade. La thioguanine (2 amino-6 mercaptopurine) et l'iododéoxyuridine sont des produits Mann. Le 2-déoxyglucose, le 2-déoxygalactose, la 5-fluorodéoxyuridine et l'arabinofuranosyl cytosine sont fournis par Sigma. L'aminoptérine provient de Schuchardt. La colchicine est fournie par le laboratoire Houde. Enfin les milieux de culture, le sérum fœtal bovin et le sérum de veau sont préparés par le laboratoire Eurobio.

\section{3. - Milieux de culture}

Les deux souches ont été initialement cultivées en tapis, en MEM tamponné Hanks (EAGLE, I959) et complété avec des acides aminés non essentiels (I0-4 $\mathrm{M}$ chacun), $4 \mathrm{p}$. Ioo de sérum foetal bovin et $6 \mathrm{p}$. Ioo de sérum de veau normal. Dans ce milieu les acides aminés non essentiels ont été remplacés par la sérine, l'asparagine et le pyruvate de sodium $\left(5^{\cdot} \mathrm{Io}^{-} 4 \mathrm{M}\right.$ chacun). Le $\mathrm{pH}$ est maintenu entre 7 et 7,4 grâce à un tampon Hanks - Tris $\mathrm{HCl}_{10} \mathbf{0}^{-2} \mathrm{M}$. Dans ces dernières conditions de culture et à une température de $36,5^{\circ} \mathrm{C}$, le temps de doublement de la souche est de I2 heures en phase exponentielle. Lorsque le milieu ne doit pas contenir les constituants dialy. sables du sérum, ce dernier est dialysé 24 heures contre l'eau courante puis contre Io fois son volume de solution saline de Hanks pendant 6 heures à $4^{\circ} \mathrm{C}$ avec un renouvellement afin de redissoudre le précipité protéique formé par baisse de la force ionique. Dans ce milieu la croissance cellulaire est plus lente $\left(T=I_{5} \mathrm{~h}\right.$ ) mais les conditions de test sont néanmoins aussi reproductibles.

La culture en suspension des deux souches peut être effectuée dans le milieu MEM (EAGLE, 1959), avec les modifications suivantes : addition de sérine, asparagine, pyruvate de sodium $\left(5 \cdot 10^{-4} \mathrm{M}\right.$ chacun) suppression du calcium, concentration dix fois plus élevée des phosphates et Tris $\mathrm{HCl}_{1} \mathrm{I}^{-2} \mathrm{M}, \mathrm{pH} 7,2$ empêchant les remontées de $\mathrm{pH}$ au cours de l'agitation. Le milieu contient 6 p. Ioo de sérum fœtal bovin dialysé ou non (le sérum de veau rend les milieux turbides). Les cultures sont effectuées en bouteilles cylindriques de $250 \mathrm{ml}$ contenant roo ml de milieu et agitées orbitalement à Ioo $\mathrm{tr} / \mathrm{mn}$. Les comptages sont effectués au Coulter Counter (précision des comptages \pm 2 p. roo). Une croissance exponentielle de 3 jours sans arrêt de croissance après dilution peut être obtenue à partir de concentrations cellulaires initiales de $\mathrm{I}$ à $2 \cdot 10^{4}$ cellules $/ \mathrm{ml}$. L'entretien de la souche S I 3 est effectuée par dilution au I/5o deux fois par semaine dans le milieu à base de sérum non dialysé. Tous les milieux de culture sont stockés congelés à - $20^{\circ} \mathrm{C}$ après addition de pénicilline (IOO UT/ml) et de streptomycine (Ioo $\mu \mathrm{g} / \mathrm{ml}$ ) et filtration sur filtre millipore GSPW 14250.

\section{4. - Étude du seuil d'action des drogues}

A l'aide d'une repipette 500 cellules sont déposées en milieu standard pour culture en tapis dans chaque bouteille de culture (flacons de $60 \mathrm{~cm}^{3}$ en verre ayant $25 \mathrm{~cm}^{2}$ de surface d'étalement). Les cellules sont incubées 6 heures, lavées deux fois avec la solution saline de Hanks, puis le milieu contenant la drogue est introduit. 8 jours après l'étalement des cellules, les colonies formées sont comptées après fixation au TCA $5 \mathrm{p}$. Ioo et coloration au bleu de Unna. Les résultats sont exprimés soit en taux de survie : rapport du nombre de colonies obtenues avec drogue au nombre de colonies obtenues en milieu sans drogue; soit en efficacité d'étalement : rapport du nombre de colonies obtenues après croissance au nombre de cellules initialement introduites. Seules les colonies visibles à l'xil nu sont comptées. Les colonies visibles au microscope et non visibles à l'œil nu sont des clones non viables ne proliférant plus dont il n'est pas tenu compte dans les comptages. Chaque concentration en drogue est testée sur 2 bouteilles identiques et chaque manipulation effectuée deux fois. (Précision des comptages $\pm 5 \mathrm{p}$. Ioo). La dose létale ıo p. Ioo est đéterminée avec une incertitude relative maximale $3^{\circ} \mathrm{p}$. Ioo sur la concentration en drogue.

\section{5. - Dosages des acides aminés des milieux de culture}

Les milieux sont déprotéinisés après addition de TCA Io $p$. Ioo et centrifugation du précipité (4 ooo $g$ pendant $50 \mathrm{mn}$ ). Le surnageant est extrait à l'éther jusqu'au retour à pH 6 afin d'éliminer le TCA et recentrifugé après extraction.

Des aliquotes de $500 \mu l$ sont analysés avec une chaîne autoanalyseur Beckman de dosage des acides aminés. Les concentrations en acides aminés dans les échantillons sont calculées par mesure de surface des pics des enregistrements. Deux chromatographies sont effectuées à partir de chaque échantillon. Elles permettent de doser la plupart des acides aminés non basiques et de séparer sérine et amides. 


\section{6. - Caryotype}

Le caryotype de la, souche $\mathrm{BHK} 2 \mathrm{I} / \mathrm{I}_{3}$ indique que cette souche possède un total de 43 à 44 chromosomes assez difficiles à distinguer et à apparier par les techniques de coloration homogène. Une méthode inspirée de WANG et FEDOROFF, (1972) utilisant une hydrolyse ménagée à la trypsine avant coloration au Giemsa a été mise au point.

Après un blocage de 4 heures par la colcémide $2 \mu \mathrm{g} / \mathrm{ml}$ les cellules sont soumises à un choc hypotonique de $4^{\circ} \mathrm{mn}$ dans le milieu suivant : sérum de veau $20 \mathrm{ml}$, eau distillée $80 \mathrm{ml}, 250$ unités de hyaluronidase (Choay) puis fixées ro $\mathrm{mn}$ dans un mélange acide acétique alcool ( $\mathrm{I} \mathrm{V}: 3 \mathrm{~V}$ ) à $4^{\circ} \mathrm{C}$.

Les lames sont hydrolysées par la trypsine $(\mathrm{o}, 2 \mathrm{~g} / \mathrm{l})$ pendant $5 \mathrm{mn}$ à $20^{\circ} \mathrm{C}$ dans le tampon suivant : chlorure de sodium $9 \mathrm{~g}$, phosphate monopotassique $7,4 \mathrm{~g}$ phosphate disodique $20,6 \mathrm{~g} \mathrm{pH} \mathrm{6,7} \mathrm{q.s.p.} \mathrm{I} 000 \mathrm{ml}$. L'hydrolyse est arrêtée par passage dans l'eau distillée puis les cellules sont colorées $7 \mathrm{mn}$ au Giemsa à 4 p. Ioo dans le tampon d'hydrolyse.

\section{RÉSULTATS}

\section{I. - Recherche d'un milieu permettant le clonage de la souche}

Les milieux MEM et I99 (MORGAN et al., I950) ne permettent pas un clonage correct de 1a souche $\mathrm{BHK}$ 2I/13 (efficacité d'étalement inférieure à $\mathrm{I}$ p. Ioo). Seul 1e milieu MEM complémenté avec les acides aminés non essentiels ( $1 \mathrm{O}^{-2} \mathrm{M}$ chacun) permet cette croissance clonale en présence de sérum foetal bovin.

\section{TABLEAU I}

\section{Efficacité d'étalement}

de la souche $S 13$ en MEM complété avec les acides aminés non essentiels et une quantité variable de sérum

I SF : I p. Ioo de sérum fotal bovin

I SV: I p. Ioo de sérum de veau

4 SFD + 6 SVD : complément sérique dialysé

\begin{tabular}{c|c|c|c|c|c|c|c|c}
\hline \hline $\begin{array}{c}\text { Sérum dans } \\
\text { le milieu (\%) }\end{array}$ & Néant & $5 \mathrm{SV}$ & $10 \mathrm{SV}$ & $4 \mathrm{SF}$ & $10 \mathrm{SF}$ & $30 \mathrm{SF}$ & $4 \mathrm{SF}+6 \mathrm{SV}$ & $4 \mathrm{SFD}+6 \mathrm{SVD}$ \\
\hline $\begin{array}{c}\text { Efficacité } \\
\text { d'étalement (\%) }\end{array}$ & 0 & 7 & 16 & 10 & 35 & 41 & 31 & 25 \\
\hline
\end{tabular}

L'addition de diverses substances dans le milieu de culture n'a pas permis d'augmenter cette efficacité d'étalement (biotine, cyanocobalamine, insuline, glutathion, acide ascorbique, acide lipoïque, taurine, acide para-amino-benzoïque, ménadione, acétate, hypoxanthine, thymidine, uridine, AMP cyclique, fer, cuivre, zinc). De même l'addition de milieux de cultures plus complexes (NCTC Iog, tryptose) n'a pas modifié cette efficacité d'étalement: seule l'addition d'une plus grande quantité de sérum fotal a eu un effet positif (tabl. I). 


\section{2. - Rôle des acides aminés non essentiels dans les conditions de clonage}

En absence d'acides aminés non essentiels l'efficacité d'étalement de la souche est inférieure à I $\mathrm{p}$. Ioo. Ces acides aminés ont été testés en milieu MEM contenant $8 \mathrm{p}$. Ioo de sérum dialysé afin de déterminer leur effet sur les conditions de clonage (tabl. 2). La sérine et l'asparagine ont un effet prépondérant (fig. I). Ces acides aminés

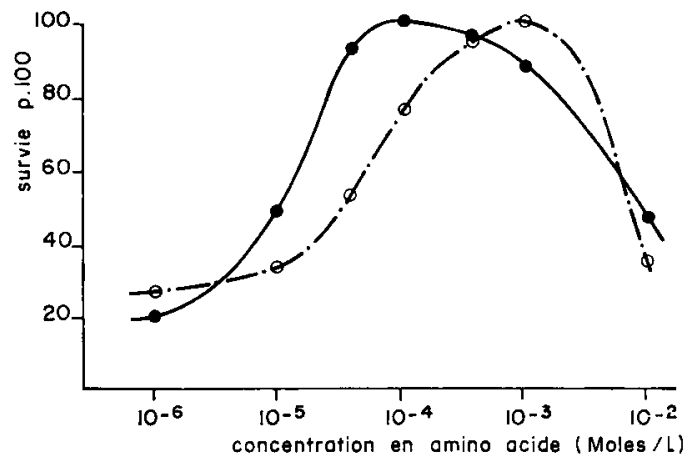

FIG. I. - Effet des concentrations en sérine et en asparagine sur l'efficacité d'étalement des $B H K 21 / 13$

Les cellules sont lavées 2 fois dans la solution saline de Hanks, 6 heures après repiquage puis les milieux suivants sont introduits.

- MEM $+{ }_{4} \mathrm{SFD}+6 \mathrm{SVD}+$ Tris $\mathrm{IO}^{-2} \mathrm{M}+$ Asparagine $5 \cdot \mathrm{ro}^{-4} \mathrm{M}+$ concentration variable en sérine.

- $\mathrm{MEM}+{ }_{4} \mathrm{SFD}+6 \mathrm{SVD}+$ Tris $1 \mathrm{IO}^{-2} \mathrm{M}+$ sérine $5 \cdot 1 \mathrm{O}^{-4} \mathrm{M}+$ concentration variable en asparagine.

Les résultats sont exprimés en taux de survie. Témoin Ioo p. Ioo de survie : MEM $+{ }_{4} \mathrm{SFD}+$ Tris $10^{-2} \mathrm{M}+$ sérine $5 \cdot 10^{-4} \mathrm{M}+$ asparagine $5 \cdot 10^{-4} \mathrm{M}$.

\section{TABLEAU 2}

Rôle des acides aminés non essentiels et du pyruvate dans la croissance clonale

Efficacité d'étalement de la souche BHK 2 I/3 I en milieu MEM contenant 8 p. Ioo de sérun fœtal bovin dialysé. Chaque métabolite est apporté à la concentration de $5 \cdot 10^{-4} \mathrm{M}$. Seuls les acides aminés jouant un rôle stimulant ont été mentionnés ici.

\begin{tabular}{|c|c|}
\hline Complérnent & $\begin{array}{c}\text { Efficacité d'étalement } \\
(\%)\end{array}$ \\
\hline$-\ldots \ldots$ & 0 \\
\hline Sérine $\ldots \ldots \ldots \ldots \ldots \ldots \ldots \ldots \ldots \ldots$ & 0 \\
\hline Asparagine $\ldots \ldots \ldots \ldots \ldots \ldots \ldots \ldots \ldots$ & 1 \\
\hline Pyruvate $\ldots \ldots \ldots \ldots \ldots \ldots \ldots \ldots \ldots$ & 0 \\
\hline Proline $\ldots \ldots \ldots \ldots \ldots \ldots \ldots \ldots \ldots \ldots$ & 0 \\
\hline Sérine + asparagine $\ldots \ldots \ldots \ldots \ldots \ldots$ & 18 \\
\hline Sérine + pyruvate $\ldots \ldots \ldots \ldots \ldots \ldots \ldots$ & 0 \\
\hline Sérine + proline $\ldots \ldots \ldots \ldots \ldots \ldots$ & 0 \\
\hline Sérine + Asparagine + Pyruvate ......... & 23 \\
\hline Sérine + Asparagine + Proline $\ldots \ldots \ldots \ldots$ & 21 \\
\hline Acides aminés non essentiels $\left(10^{-2} \mathrm{M}\right.$ chacun $) \ldots$ & 27 \\
\hline
\end{tabular}


ne sont cependant pas essentiels à la croissance puisqu'à forte densité cellu1aire les BHK 2r/23 poussent correctement en MEM non complémenté avec les acides aminés " non essentiels".

Le pyruvate de sodium semble assurer une meilleure reproductibilité des manipulations de clonage : son rôle stimulant est particulièrement net lorsque les cellules ont été malmenées au cours du repiquage (passage trop long à $20^{\circ} \mathrm{C}$, tapis resté trop longtemps en milieu acide, cellules trop trypsinées). Dans ce cas la présence du pyruvate de sodium restaure une efficacité d'étalement normale.

\section{3. - Rôle des acides aminés non essentiels dans la croissance en suspension des $S 13$}

La souche $\mathrm{S} \mathrm{I}_{3}$ est usuellement entretenue à des concentrations cellulaires supérieures à 200000 cellules/m1. Dans ces conditions, la phase de croissance exponentielle est très courte, de durée inférieure à 24 heures. Dans le milieu MEM 6 SE modifié pour la culture en suspension, la croissance à une concentration cellulaire inférieure à Ioo ooo cellules $/ \mathrm{ml}$ s'est avérée très irrégulière. L'addition de sérine et d'asparagine $\left(5 \cdot \mathrm{IO}^{-4} \mathrm{M}\right)$ ou de l'ensemble des acides aminés non essentiels $\left(I^{-2} \mathrm{M}\right)$ dans ce milieu de culture permet d'obtenir de façon reproductible des croissances exponentielles d'une durée de 72 heures avec une concentration cellulaire initiale de ro ooo cellules/ml (fig. 2).

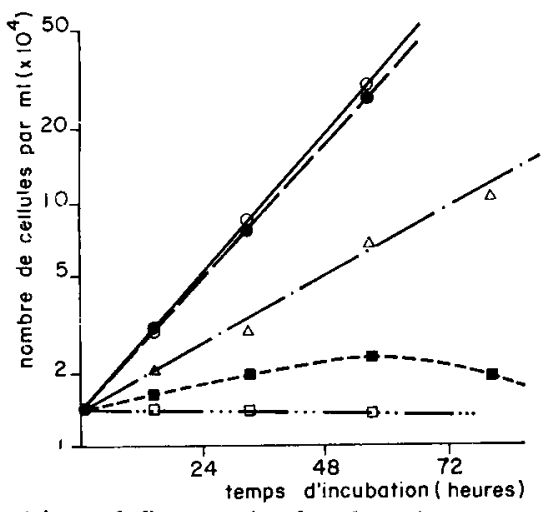

FIG. 2. - Rôle de la sérine et de l'asparagine dans la croissance en suspension des $S 13$

Une culture de cellules en fin de phase exponentielle est concentrée par centrifugation (Ioo g; $5 \mathrm{mn}$ ) à $5^{\circ} \cdot \mathrm{O}^{6} \mathrm{c} / \mathrm{ml}$. Les cellules sont ensuite diluées à $\mathrm{I}, 5^{\circ} 10^{4} \mathrm{c} / \mathrm{ml}$ en $\mathrm{MEM}_{4} \mathrm{SFD}$, Tris $\mathrm{IO}^{-2} \mathrm{M}$, phosphates $5 \cdot 10^{-3} \mathrm{M}$, sans calcium, complété comme suit :

口 sans complément

$0+$ acides aminés non essentiels $\mathbf{1 0}^{-2} \mathrm{M}$ chacun

- + sérine $5 \cdot 10^{-4} \mathrm{M}+$ asparagine $5 \cdot 10^{-4} \mathrm{M}$

- asparagine $5 \cdot 10^{-4} \mathrm{M}$

$\Delta+$ sérine $5 \cdot 10^{-4} \mathrm{M}$

La croissance en suspension en milieu à base de sérum dialysé est possible dans les mêmes conditions (MEM $+6 \mathrm{SED}+$ sérine et asparagine $5 \cdot \mathrm{Io}^{-4} \mathrm{M}$ ) cependant une série de repiquages dans ce même milieu induit une décroissance progressive du rythme de division cellulaire.

La souche $\mathrm{BHK} 2 \mathrm{I} / \mathrm{I} 3$ non adaptée à la culture en suspension prolifère aussi dans ces conditions mais son plateau de croissance est bas $\left(3 \cdot \mathrm{IO}^{5}\right.$ cellules $\left./ \mathrm{ml}\right)$ alors que la souche $\mathrm{S}$ I3 prolifère jusqu'à la concentration de Io cellules $/ \mathrm{ml}$ ). 


\section{4. - Épuisement du milieu de culture et arrêt de croissance}

La composition du milieu de culture ne se modifie pas de façon similaire à faible densité de population et en culture confluente (tabl. 3).

Dans les conditions de clonage une quantité importante d'alanine est libérée par les cellules. A forte concentration, l'alanine inhibe la croissance si elle est le seul acide aminé non essentiel introduit dans le milieu de culture. Cet amino-acide est peut-être responsable de la diminution de l'efficacité d'établement en milieu préconditionné dans les conditions de clonage (OFLLERMAN et MILLER, I969). Dans les conditions de confluences un certain nombre de métabolites disparaissent du milieu de culture; sérine, asparagine et glutamine. D'autres voient leur concentration sérieusement diminuée : méthionine, leucine et isoleucine. D'autres enfin apparaisraissent : glycine et proline. La glutamine et l'asparagine ne disparaissent pas par simple désamination car les acides glutamiques et aspartiques n'apparaissent pas dans le milieu de façon notable. L'asparagine étant non essentielle à la croissance à forte densité de population, 1'arrêt de croissance peut être imputé à une carence en glutamine.

\section{TABLEAU 3}

Concentration en acides aminés

dans le milieu de culture en fonction des conditions de croissance

Le3 résultats sont exprimés en multiples de $10^{-4} \mathrm{M}$; les tirets correspondent à des valeurs inférieures à $1 \mathrm{O}^{-5} \mathrm{M}$.

Témoin : milieu MEM complémenté avec sérine asparagine et pyruvate $5 \cdot 10^{-4} \mathrm{M}, 4 \mathrm{p}$. Ioo de sérum fotal bovin dialysé et $6 \mathrm{p}$. Ioo de sérum de veau dialysé.

Clonage : le même milieu est successivement passé pendant $7^{2}$ heures sur trois cultures de cellules étalées en conditions de clonage (I0o cellules $/ \mathrm{cm}^{2}$ ). Au troisième passage l'efficacité d'étalement est très diminuée (3 p. Ioo).

Confluence : même manipulation pour les conditions de confluence $\left(5 \cdot \mathrm{IO}^{4} \mathrm{cellules} / \mathrm{cm}^{2}\right)$ le $\mathrm{pH}$ est périodiquement ramené à 7,2 (dans ces conditions les cellules peuvent croître sans sérine et sans asparagine). Au troisième passage les cellules ne prolifèrent plus.

\begin{tabular}{|c|c|c|c|}
\hline Acide aminé & Témoin & Clonage & Confluence \\
\hline A. aspartique $\ldots \ldots \ldots$ & 0,1 & - & $0,1 \cdot$ \\
\hline A. glutamique $\ldots \ldots \ldots \ldots$ & 0,2 & 0,2 & 一 \\
\hline Glycine $\ldots \ldots \ldots \ldots \ldots$ & - & 2,0 & 4,9 \\
\hline Alanine $\ldots \ldots \ldots \ldots \ldots$ & 一 & 5,8 & 0,2 \\
\hline Sérine $\ldots \ldots \ldots \ldots \ldots$ & 4,0 & 2,5 & 0,1 \\
\hline Proline . . . . . . . . . . & - & 0,6 & 2,1 \\
\hline Asparagine + glutamine . & 16,2 & 7,4 & - \\
\hline Méthionine $\ldots \ldots \ldots \ldots$ & 1,2 & 0,8 & 0,2 \\
\hline Isoleucine $\ldots \ldots \ldots \ldots \ldots$ & 3,8 & 2,5 & 0,6 \\
\hline Leucine . . . . . . . . . & 3,8 & 2,6 & 0,8 \\
\hline
\end{tabular}

Ce fait est confirmé dans les conditions de culture en suspension : le plateau d'arrêt de croissance est élevé par addition de glutamine ; il n'est pas élevé par addition de glucose, de leucine, d'asparagine, de sérine, et de lysine ou par addition de sérum. 


\section{5. - Effet de pH sur la croissance et l'efficacité d'étalement}

Le rythme de division cellulaire est maximum à $\mathrm{pH}$ 7,I (fig. 3). La souche supporte mal les passages à $\mathrm{pH}$ acide (milieux épuisés) surtout dans les conditions de culture en suspension. L'efficacité d'étalement optimale est obtenue à $\mathrm{pH}$ 7,6 et décroît fortement aux $\mathrm{pH}$ acides. Les effets de $\mathrm{pH}$ sur l'efficacité d'étalement et le rythme de croissance de la souche dépendent de la nature du sucre introduit dans le milieu de culture. En présence de galactose les cellules ont une efficacité d'étalement optimale à $\mathrm{pH} 7,0$ et un rythme de croissance maximum à $\mathrm{pH} 6,9$. En présence de glucose les conditions de croissance optimales sont donc nettement plus alcalines que dans un milieu à base de galactose.

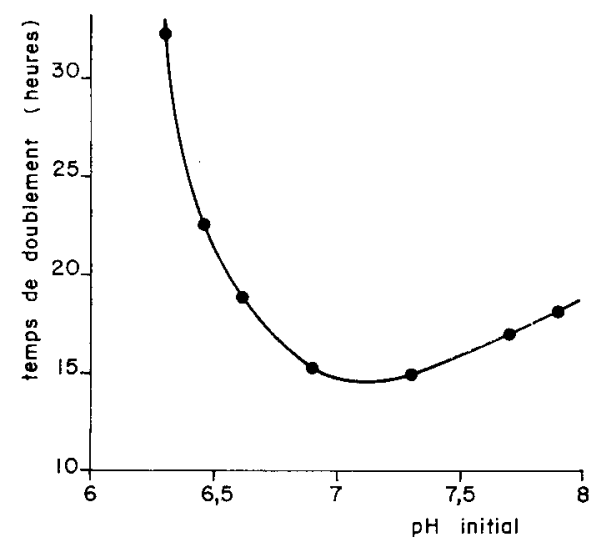

Fig. 3. - Effet du pH sur le rythme de division des $S 13$ cultivées en suspension

Les cultures sont effectuées en milieu standard. A l'aide d'acide chlorhydrique ou de soude (o, I N), le milien est amené au $\mathrm{pH}$ désiré au moment du repiquage. Avec une concentration cellulaire initiale de $1,5 \cdot 10^{4} \mathrm{c} / \mathrm{ml}$ les variations d'acidité enregistrées ne dépassent pas $0, \mathbf{I}$ unité $\mathrm{pH}$ au cours des premières 48 heures.

\section{6. - Effet de la température sur la croissance et l'efficacité d'étalement}

L'efficacité d'étalement de la souche $\mathrm{BHK} 2 \mathrm{I} / \mathrm{I}_{3}$ est constante de $33^{\circ} \mathrm{C}$ à $3^{\circ} \mathrm{C}$ et chute brutalement à $39^{\circ} \mathrm{C}$, lorsque les cellules sont étalées en milieu standard à base de sérum non dialysé. Dans les mêmes conditions de test la croissance n'est ralentie qu'au-dessous de $34^{\circ} \mathrm{C}$ ou au-dessus de $38,8^{\circ} \mathrm{C}$ (fig. 4).

En milieu à base de sérum dialysé l'efficacité d'étalement décroît graduellement pour les températures inférieures à $36,5^{\circ} \mathrm{C}$. Cette décroissance n'est pas due à l'apparition d'un besoin supplémentaire en acides aminés non essentiels ou en bases puriques et pyrimidiques. Aux températures supérieures à $39^{\circ} \mathrm{C}$ les cellules deviennent géantes, prenant un aspect semblable à des cellules dont la synthèse d'ADN est inhibée.

\section{7. - Seuil de résistance à diverses drogues (tabl. 4)}

L'aminoptérine est toxique à très faible concentration ( $\left(\mathrm{IO}^{-8} \mathrm{M}\right)$; son effet peut être réversé par le mélange hypoxanthine $\left(\mathrm{IO}^{-4} \mathrm{M}\right)$, thymidine $\left(\mathrm{IO}^{-5}\right)$ et glycine $\left(5 \cdot 10^{-4} \mathrm{M}\right)$. L'étude des seuils de résistance à l'aminoptérine en l'absence d'un stul 


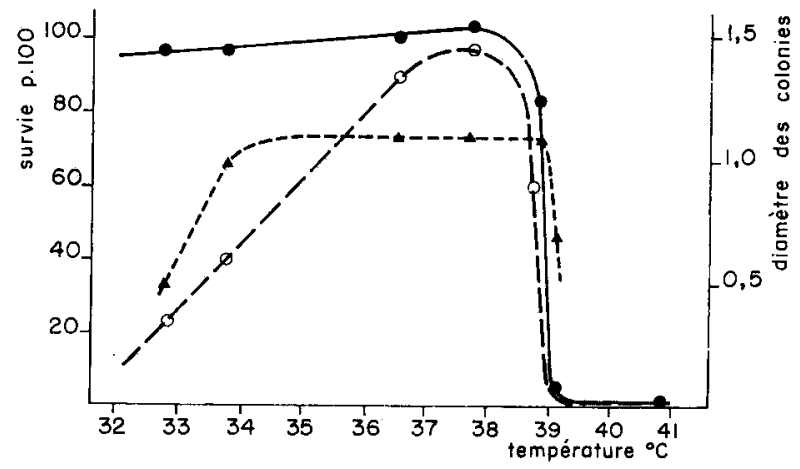

FIG. 4. - Effet de la température sur la croissance et l'efficacité d'étalement des BHK 21/13

Les températures sont estimées par mesure directe dans le milieu de culture 3 fois au cours de la manipulation à l'aide d'une microsonde (fluctuation $\pm 0,15^{\circ} \mathrm{C}$ ).

- survie en milieu standard, sérum non dialysé.

o survie en milieu standard, sérum dialysé.

- Diamètre moyen des colonies en unités arbitraires.

\section{TABLEAU 4}

\section{Seuils de résistance aux drogues de la souche $B H K 21 / 13$}

Les cellules sont testées dans les conditions d'efficacité d'étalement en milieu MEM tamponné Hanks $t$ ris $\mathrm{I}^{-2} \mathrm{M}$ complémenté avec sérine, asparagine et pyruvate de sodium $5^{\cdot} 1 \mathrm{O}^{-4} \mathrm{M}, 4 \mathrm{p}$. 100 de sérum fœtal et $6 \mathrm{p}$. Ioo de sérum de veau dialysé (D) ou non dialysé (ND). Les résultats sont exprimés en concentration en drogue donnant lieu à une survie de Io p. Ioo par rapport au témoin non traité ( $\mathrm{DL}_{10}$ ou dose létale Io $\mathrm{p}$. IOo). La croissance s'effectue en présence de drogue jusqu'au comptage des colonies.

\begin{tabular}{|c|c|c|}
\hline Drogue & $\begin{array}{l}\text { Complément } \\
\text { sérique du } \\
\text { milieu }\end{array}$ & $\mathrm{DL}_{\mathbf{1 0}}$ \\
\hline 5-bromodeoxyuridine .... & ND & $4 \cdot 10^{-6} \mathrm{M}$ \\
\hline 5-iododéoxyuridine $\ldots .$. & ND & $2 \cdot 10^{-5} \mathrm{M}$ \\
\hline 5-fluorodéoxyuridine $\ldots \ldots \ldots \ldots \ldots$ & $\mathrm{D}$ & $4 \cdot 10^{-9} \mathrm{M}$ \\
\hline 5 -fluorodéoxyurudine + thymidine $10^{-5} \mathrm{M}$ & $\mathrm{D}$ & $10^{-6} \mathrm{M}$ \\
\hline Thymidine $\ldots \ldots \ldots \ldots \ldots \ldots \ldots \ldots$ & $\mathrm{D}$ & $4 \cdot 10^{-4} \mathrm{M}$ \\
\hline Aminoptérine..$\ldots \ldots \ldots \ldots \ldots \ldots$ & $\mathrm{D}$ & $1,5 \cdot 10^{-8} \mathrm{M}$ \\
\hline Aminoptérine $+\mathrm{H}+\mathrm{G}\left({ }^{1}\right) \ldots \ldots \ldots \ldots$ & $\mathrm{D}$ & $6 \cdot 10^{-8} \mathrm{M}$ \\
\hline Aminoptérine $+\mathrm{H}+\mathrm{T} \ldots \ldots$. & $\mathrm{D}$ & $1,5 \cdot 10^{-8} \mathrm{M}$ \\
\hline Aminoptérine $-\mathrm{G}+\mathrm{T} \ldots \ldots \ldots \ldots$ & $\mathrm{D}$ & $1,5 \cdot 10^{-8} \mathrm{M}$ \\
\hline Aminoptérine $+\mathrm{H}+\mathrm{G}+\mathrm{T} \ldots \ldots \ldots$ & $\mathrm{D}$ & $10^{-4} \mathrm{M}$ \\
\hline Colchicine $\ldots \ldots \ldots \ldots \ldots \ldots \ldots \ldots$ & ND & $10^{-6} \mathrm{M}$ \\
\hline Colcémide $\ldots \ldots \ldots \ldots \ldots \ldots \ldots$ & ND & $2,5 \cdot 10^{-6} \mathrm{M}$ \\
\hline 8-azaguanine $\ldots \ldots \ldots \ldots \ldots \ldots \ldots$ & ND & $3 \cdot 10^{-5} \mathrm{M}$ \\
\hline Thioguanine $\ldots \ldots \ldots \ldots \ldots \ldots \ldots$ & $\mathrm{ND}$ & $2 \cdot 10^{-7} \mathrm{M}$ \\
\hline Arabinofuranosyl cytosine . . . . . . . . & ND & $4 \cdot 10^{-7} \mathrm{M}$ \\
\hline 2-déoxyglucose + galactose $10^{-8} \mathrm{M}(2) \ldots \ldots$ & $\mathrm{D}$ & $2 \cdot 10^{-4} \mathrm{M}$ \\
\hline 2-déoxygalactose + glucose $10^{-3} \mathrm{M} \ldots \ldots$ & $\mathrm{D}$ & $5 \cdot 10^{-4} \mathrm{M}$ \\
\hline
\end{tabular}

(1) $\mathrm{H}=$ hypoxanthine $10^{-4} \mathrm{M} ; \mathrm{T}=$ Thymidine $10^{-5} \mathrm{M} ; \mathrm{G}=\mathrm{Gly}-$ cine $5 \cdot 10^{-4} \mathrm{M}$.

$\left({ }^{2}\right)$ Pour le test des analogues de sucres, le milieu ne contient pas initialement de glucose. 
de ces trois métabolites met en évidence deux niveaux de sensibilité à l'aminoptérine.

La souche résiste mieux à l'action antimitotique de la colchicine et de la colcémide que les autres souches de cellules de mammifères. Ce fait a déjà été constaté chez le hamster syrien (BAIIS, I968). La sensibilité de la souche au 2-déoxygalactose indique que le galactose peut être utilisé compétitivement au glucose. Les analogues classiques de la thymidine et de la guanine sont toxiques à faible concentration et pourront donc être employés dans les sélections de mutants résistants.

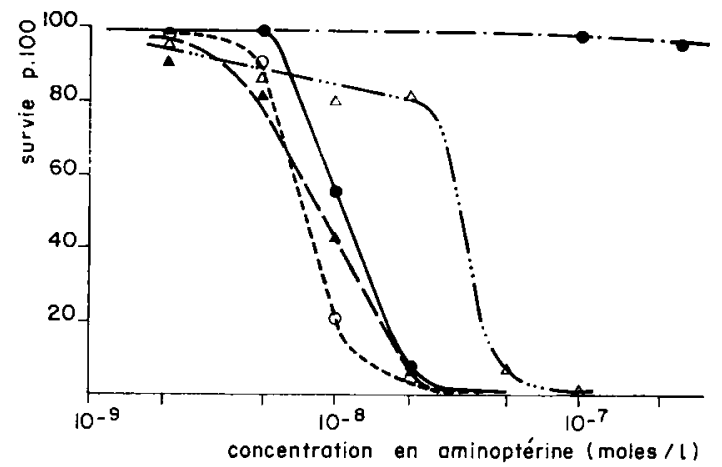

FIG. 5. - Sensibilité des BHK 21/13 à l'aminoptérine

I.es tests sont effectués en milieu MEM Tris $\mathrm{IO}^{-2} \mathrm{M}, 4 \mathrm{SFD}+6 \mathrm{SVI}$, contenant sérine, asparaginc ct pyruvate (5. $10^{-4} \mathrm{M}$ chacun). L'efficacité d'étalement en présence d'aminoptérine a concentratiorı v.ariable est testée dans les conditions suivantes :

- aucun complément

- hypoxanthine $10^{-4} \mathrm{M}+$ glycine $5 \cdot 10^{-4} \mathrm{M}+$ thymidine $10^{-5} \mathrm{M}$

- glycine $5 \cdot 10^{-4} \mathrm{M}+$ thymidine $10^{-5} \mathrm{M}$ (Carence en hypoxanthine)

$\Delta$ hypoxanthine $10^{-4} \mathrm{M}+$ thymidine $\mathrm{ro}^{-5} \mathrm{M}$ (carence en glycine)

$\Delta$ hypoxanthine $10^{-4} \mathrm{M}+$ glycine $5 \cdot 10^{-4} \mathrm{M}$ (carence en thymidine)

En présence des trois métabolites l'aminoptérine est très peu toxique à to ${ }^{-5} \mathrm{M}$.

\section{8. - Caryotype}

Le hamster syrien a un caryotype constitué de 44 chromosomes. La souche BHK 2I/I3 possède aussi ce même nombre de chromosomes (FISHER et al., I97I). Après clonage en milieu à base de sérum dialysé nous avons obtenu une souche ayant un nombre modal de 43 chromosomes (tabl. 5).

\section{TABLEAU 5}

Fréquence du nombre de chromosomes par métaphase

La souche a été clonée en milieu à base de sérum dialysé et son caryotype effectué après 20 divisions dans ce milieu

\begin{tabular}{l|c|c|c|c|c|c}
\hline \hline Nombre de chromosomes & 41 & 42 & 43 & 44 & 45 & $n$ \\
\hline $\begin{array}{c}\text { Pourcentage dans la } \\
\text { population en métaphase }\end{array}$ & 1 & 8 & 62 & 19 & 5 & 3 \\
\hline
\end{tabular}



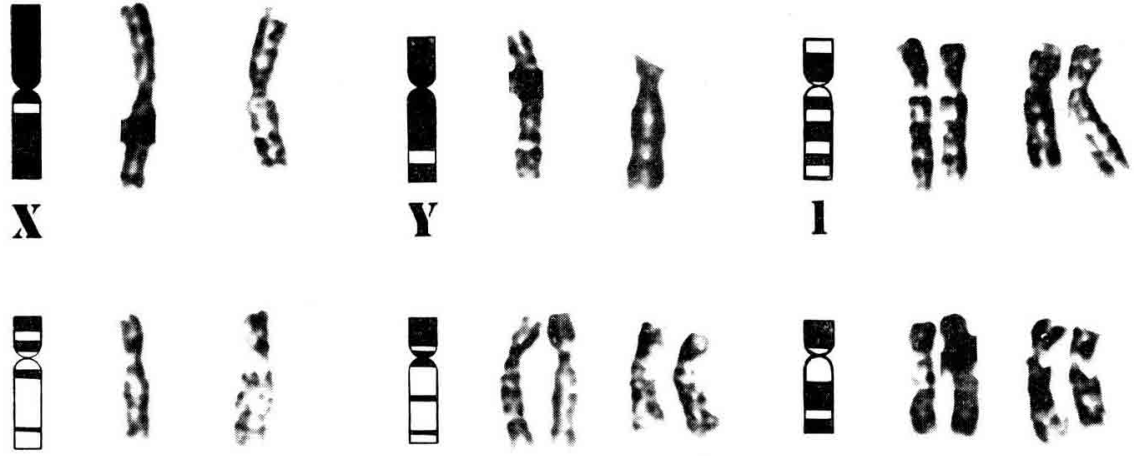

2

3

4

- 5

音
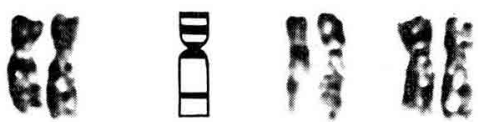

5

(i)

\%

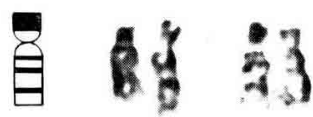

羿

ำ

8

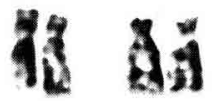

9

10

11

ㅁำ ถูก

요

旦

是 5 .

12

13

14

$8 \quad x) \quad 8$

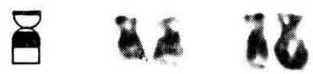

员 $20 . x \sin$

15

20

21

目

960 敌

目 能的

16

18

18

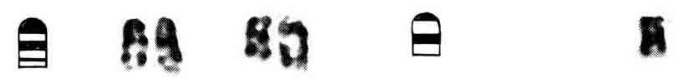

\section{BHK21C13}

1)

$\mathbf{R}$

FIG. 6. - Caryotype à bandes de la souche BHK 21/13 clonée en milieu à base de sérum dialysé

Deux métaphases caractéristiques, ayant 43 chromosomes chacune, sont analysées. L'une d'elles est trisomique pour le chromosome ro, l'autre possède un chromosome remanié (R). La paire 8 est absente dans les caryotypes analysés.

La densité de population cellulaire et le degré d'étalement des chromosomes influent sur les conditions d'hydrolyse, rendant la méthode assez délicate a employer de façon reproductible. 
La technique de coloration après protéolyse ménagée permet de distinguer toutes les paires de chromosomes les unes des autres. La souche possède un caryotype mâle. En plus des chromosomes $\mathrm{X}$ et $\mathrm{Y}$, un chromosomes est présent à un seul exemplaire $\left(n^{\circ} 2\right)$. Une paire est absente (paire $\left.n^{\circ} 8\right)$. Enfin la souche est trisomique pour le chromosome 21 . Sur les 43 chromosomes du caryotype, 42 sont donc retrouvés régulièrement dans les métaphases, le chromosome supplémentaire pouvant varier (trisomie sur le chromosome to ou chromosome acrocentrique de petite taille).

L'aspect des chromosomes correspond assez bien aux observations faites par MARSHALL (I972) : les bandes sombres observées ici correspondent aux zones fluorescentes apparaissant après le traitement à la quinacrine (fig. 6). La comparaison des caryotypes a permis d'identifier le chromosome $\mathrm{Y}$.

\section{DISCUSSION}

La mise au point de techniques de sélection de mutants du métabolisme intermédiaire chez $\mathrm{BHK} 2 \mathrm{I} / \mathrm{I} 3$ nécessite une connaissance préalable des caractéristiques principales de cette souche (efficacité d'étalement, besoins nutritionnels, sensibilité à diverses drogues, caryotype).

L'efficacité d'étalement de la souche ne dépasse pas $40 \mathrm{p}$. Ioo dans les meilleures conditions obtenues. Plusieurs hypothèses peuvent expliquer ce résultat:

- Le milieu de culture contient un métabolite en quantité excessive (OELLERMAN et MLLLER, 1969).

- un ou plusieurs métabolites supplémentaires sont nécessaires à une croissance optimale de la souche (HAM, I965).

- Les conditions de repiquage (trypsination et dissociation) sont trop sévères (Puck, 1956).

- Enfin la quantité de sérum introduite dans le milieu est insuffisante bien que déjà élevée (Io p. IOO). 5 p. Ioo de sérum dans le milieu de culture permettent une initiation correcte de la synthèse d'ADN (CrARKE et al., I970). Le sérum semble perdre un facteur de croissance par dialyse sans diminution importante de l'efficacité d'étalement. Le sérum intervient donc a plusieurs niveaux dans la croissance clonale : il apporte les globulines nécessaires à l'attachement et l'initiation du cycle cellulaire (FIShER et al., I959); (HEAly et PARKer, I968); il apporte simultanément un ou plusieurs facteurs de croissance dialysables (ME'TZER et MosKOWITZ, I960 ; HOLLEY et KIERMAN, I968).

La sérine, 1'asparagine et le pyruvate de sodium sont nécessaires à la croissance clonale de certaines souches (EAGLE et PIEz, I962; OelLLermain et MILLER, I969). En particulier la souche BHK 2I/13 ne prolifère dans les conditions de clonage qu'en présence d'une concentration en sérine supérieure à $10^{-5} \mathrm{M}$ et une concentration en asparagine supérieure à $5 \cdot \mathrm{IO}^{-5} \mathrm{M}$. Aux fortes concentrations cellulaires 1a croissance devient possible en absence de ces métabolites car ils sont rejetés par les cellules dans le milieu et s'y accumulent à des concentrations permettant la croissance. La présence de sérine et d'asparagine permet de même la croissance en suspension des $\mathrm{S}$ I3 à une très faible densité cellulaire (jusqu'à $I O^{3}$ cellules $/ \mathrm{m} 1$ ); de 
nombreuses souches cellulaires prolifèrent difficilement au-dessous d'une concentration cellulaire inférieure à $10^{5}$ cellules $/ \mathrm{ml}$; on peut supposer qu'un milieu permettant le clonage de ces souches permettra leur culture en suspension à faible densité de population.

Plusieurs facteurs interviennent dans l'arrêt de croissance à forte concentration cellulaire : acidité du milieu (CECCARINI et EAGLE, I97I), épuisement en glutamine et en glucose (BLACKER et al., r97r), enfin accumulation de métabolites devenant ainsi toxiques (proline et alanine). Les cellules s'accumulent en phase $\mathrm{G}_{1}$ par inhibition de contact lorsqu'elles arrivent à confluence (OREN et KOHN I969); cette accumulation est certainement favorisée par la disparition de la glutamine et d'une grande partie de l'isoleucine du milieu de culture ( $L E Y$ et TOBEY, I970).

$\mathrm{La}$ souche est sensible à des concentrations très faibles d'aminoptérine $\left(\mathrm{IO}^{-8} \mathrm{M}\right)$ en milieu à base de sérum dialysé. L'existence de deux seuils distincts de résistance partielle à l'aminoptérine pour la carence en thymidine ou en hypoxanthine est à rapprocher des résultats de ORKIN et LITTLLEFIELD (I97I). Les BHK 2I/I3 peuvent proliférer en présence d'aminoptérine si le milieu de culture contient en outre le mélange glycine, hypoxanthine, thymidine (LITTLEFIEL, I963). La souche possède donc une thymidine kinase et une hypoxanthine-guanine-phosphoribosyl-transférase. Ces caractéristiques sont révélées par ailleurs par la sensibilité de la souche à l'action de la 5-bromodéoxyuridine et l'iododéoxyuridine d'une part et de la 8-azaguanine et de la thioguanine d'autre part.

Le caryotype de la souche clonée montre un certain nombre de modifications par rapport au caryotype initial des BHK 2I/13 (CAPSTICK et al., I962). La diminution progressive du nombre des chromosomes (de 44 à 43 ) au cours de la culture de la souche serible se confirmer (MARSHALI, I97I). La modification du caryotype a certainement pour origine les nouvelles conditions de culture employées pour 1'entretien de la souche. De telles modifications, liées aux conditions de culture, ont été observées sur certaines souches de porc (ECHARD, 1972).

L'existence d'un caryotype stable, relativement peu remanié et dont on peut distinguer chaque paire de chromosomes, permet de considérer la souche BHK 21/13 comme un excellent outil d'étude génétique de l'hérédité des mammifères, la mise au point des conditions de clonage de cette souche en milieu à base de sérum dialysé permet d'étudier le mode d'action de diverses drogues et en particulier de de sélectionner différents types de mutants résistants à ces drogues avec ou sans mutagenèse préalable. Des travaux d'isolement et de caractérisation de résistants à la 8-azaguanine, à la 5-bromodéoxyuridine, à l'aminoptérine, à la colchicine et au 2-déoxygalactose sont en cours. L'existence d'un certain nombre de particularités métaboliques de la souche (résistance partielle à la colchicine et à la colcémide, besoins en asparagine), la prédisposent à être employée dans les hybridations interspécifiques avec des lignées de porc pour l'étude des groupes de liaison de cet animal. La croissance clonale de la souche est possible en absence de proline, glycine, alanine, acides aspartique et glutamique. La sélection d'auxotrophes pour l'un quelconque de ces aminoacides est donc possible (KAO et PuCk, I968). Sa mise au point est effectuée. 


\section{REMERCIEMENTS}

Nous remercions Melle Yvette CApdivila du Laboratoire de Physiologie végétale de la Faculté des Sciences de Toulouse qui a effectué les chromatographies d'acides aminés ainsi que le professeur Zalta de l'Institut de Physiologie de la Faculté des Sciences de Toulouse dont les critiques nous ont été précieuses pour la préparation du manuscrit.

\section{SUMMARY}

\section{CLOWNING CONDITIONS AND SUSPENSION GROWTH \\ OF THE SYRIAN HAMSTER'S STRAIN BHK 2I/I3. STRAIN SENSIBILITY TO DRUGS AFFECTING NUCLEIC ACID METABOLISM}

Because of its characteristics, the strain $B H K 2 I / I_{3}$ is easily used as a genetic tool. Its growth and cloning conditions are here studied. The plating efficiency of the strain is low (I p. Ioo), when cultivated in Eagle's Minimum Essential Medium containing 8 p. roo of dialyzed fœtal calf serum. If serine and asparagine are added, a satisfactory clonal growth is obtained (25 p. roo of plating efficiency). In the presence of these two amino acids BHK $2 \mathrm{I} / \mathrm{I}_{3} \mathrm{~S} \mathrm{I}_{3}$ a line adapted to suspension growth, develops with a fourteen hours doubling time at low cell population density $\left(\mathrm{IO}^{4}\right.$ cells $\left./ \mathrm{ml}\right)$. Medium composition evolves during the growth of $\mathrm{BHK} 2 \mathrm{I} / \mathrm{I} 3$ cells : great quantities of alanine, glycine and proline are transferred in the medium at low cell population density. Confluent layers consume medium amides. Optimal cloning conditions are obtained at $\mathrm{pH} 7,2$ and $37^{\circ} \mathrm{C}$.

The strain sensibility to drugs affecting nucleic acids metabolism is studied (bromodeoxyuridine, azaguanine, aminopterin).

The karyotype of the strain $(\bar{n}=43)$ is observed after trypsin treatment and Giemsa staining and compared to the syrian hamster karyotype $(\bar{n}=44)$. Each pair of chromosomes is distinguished by this method.

\section{RÉFÉRE,NCES BIBLIOGRAPHIQUES}

Balis M. E., I968. Antagonists and nucleic acids. North-Holland publishing company Amsterdam, 57-58.

Birnbors M. C., I970. Optimal conditions for counting of precipited 3 H-RNA on glass fiber filters. Analyt. Biochem. 37, I78-182.

Blaker G. J., Birch J. R., Pirt S. J., I97r. The glucose insulin and glutamine requirements of suspension cultures of Hela cells in a defined culture medium. J. Cell. Sci. 9, 529-537.

Capstick P. B., Telling R. C., Chapman W. G., Stewart D. L.,' ig62. Growth of a cloned strain of hamster kidney cells in suspended cultures and their susceptibility to the virus of foot and mouth disease. Nature, 195, II63-II64.

Ceccarini C., Eagle H., r97r. pH as a determinant of cellular growth and contact inhibition. Proc. Nat. Acad. Sci., 68, 229-233.

Clarke G. D., Stoker H. G. P., Ludlow A., Thornton M., rg7o. Requirements of serum for DNA synthesis in BHK 2 I cells. Effect of density, suspension and virus transformation. Nature, 22\%, $789-801$.

EAGIE H., I959. Amino acids metabolism in mammalian cell cultures. Sciences 130, 432-437.

EAGLe H., PIEZ K., I962. The population dependent requirement by culture mammalian cells for metabolites wich they can synthesize. J. Exp. Med. 116, 29-43.

Echard G., 1973. Étude des bandes chromosomiques du porc et de trois différentes souches de rein de porc en culture (PK I $_{5}, \mathrm{~F}$ et RP). Ann. Génét. Sélect. anim. 5 (sous presse). 
Fischer A. B., Manojlovic N., Kumbert E., Beck E. G., I97I. Zytogenetische charakterisierung der zillinien BHK 2I C I3 und BHK 2I C I3 S. Arch. Virusforsch, 33, 375-843.

Fisher H. W., Puck T. T., Sato G., 1959. Molecular growth requirements of single mammalians cells III. Quantitative colonial growth of single $S_{s}$ cells in medium containing synthetic small molecular constituents and two purified fractions. J. Exp. Med. 109,649-659.

HAM R. G., I965. Clonal growth of mammalian cells in chemically defined synthetic medium. Proc. Nat. Acad. Sci. 53, 288-93.

Healy G. M., Parker R. C., I968. Cultivation of mammalian cells in defined media with protein and non protein supplements. J. Cell. Biol. 50, 539-553

Holley R. W., Kierman Y. A., 1968. "Contact inhibition " of cell division in 3 T 3 cells. Proc. Nat. Acad. Sci. 60, 300-304.

LEY K. D., Tовеy R. A., I970. Regulation of initiation of DNA synthesis in chinese hamster cells II. Induction of DNA synthesis and division by isoleucine and glutamine in $G_{1}$ arrested cells in suspension culture. J. Cell. Biol. 47, 453-459.

Littlefield W. J., I963. The inosinic acid pyrophosphorylase activity of mouse fibroblasts partially resistants to 8-azaguanine. Proc. Nat. Acad. Sci. 50, 568-573.

Mac Pherson I., Stocker, 1962. Polyma transformation of hamster cell clones an investigation of genetic factors affecting cell competence. Virology, 16, 147-15I.

Marshall R., I972. Karyotype of the BHK 2 I C 13 cell line of the syrian hamster determined with the aid of quinacrine staining. Chromosoma, 37, 395-404

Metzgar D. P., Moskowitz M., I960. Separation of growth promoting activity from horse serum by dialys. Proc. Soc. Exp. Biol. Med. 104, 363-365.

Morgan J. F., Morton H. J., Parker R. C., I950. Nutrition of animal cells in tissue culture. I. Intitial studies on a synthetic medium. Proc. Soc. Exp. Biol. Med., 173, I-I2.

Oellerman R. A., Milier E. M., I969. The influence of conditionned media and non essential aminoacid supplementation on the growth of cells in, vitro. J. Cell. Physiol., 74, 299-306.

OREN R., KoHN A., I969. Density dependent inhibition of cell growth in cultures of primary and established lines of cells. I. Cells Physiol., 74, 307-3I4.

Puck T. T., Marcus P. I., Cieriura S. J., 1956. Clonal growth of mammalian cells in vitro. Growth characteristics of colonies from single Hela cells with and without a feeder layer. J. Exp. Med. 108, 273-283.

Stocker M., Mac Pherson I., 1964. Syrian fibroblast cell line BHK 21 and its derivates. Nature, 203, I355-I357.

Todar G. J., Aaronson S. A., Rands E., I97I. Rapid detection of mycoplasma infected cultures. Expt. Cell. Res., 65, 256-257.

WANG H., FEDOROFF S., 1972. Banding in human chromosomes treated with trypsin. Nature New biology, 235, 52-54.

\section{ANNEXE}

\section{ABRÉVIATIONS}

\begin{tabular}{r|l} 
ADN & Acide déoxyribonucléique \\
BHK & Baby Hamster Kidney \\
EDTA & Acide éthylène diaminetétraacétique \\
MEM & Minimum Essential Medium (EAGLE) \\
SF & Sérum foetal bovin \\
SV & Sérum de veau \\
SVD & Sérum de veau dialysé \\
TCA & Acide trichloroacétique \\
Tris & trishydroxyméthyleaminométhane
\end{tabular}

